DOI: $10.1515 /$ awutp -2015-0208

\title{
CLOUDS AND THE NEAR-EARTH ENVIRONMENT: POSSIBLE LINKS
}

\author{
Simona Condurache-Bota ${ }^{*}$, Mirela Voiculescu, Carmelia Dragomir \\ Dunarea de Jos University of Galati, Faculty of Sciences and Environment, Chemistry, \\ Physics and Environment Department, 111 Domneasca Street, 800201 Galati, Romania. \\ *Corresponding author: scondurache@ugal.ro
}

\begin{abstract}
Article Info
Received: 04.02.2015

Accepted: 11.05.2015
\end{abstract}

Keywords: Clouds

\begin{abstract}
Climate variability is a hot topic not only for scientists and policy-makers, but also for each and every one of us. The anthropogenic activities are considered to be responsible for most climate change, however there are large uncertainties about the magnitude of effects of solar variability and other extraterrestrial influences, such as galactic cosmic rays on terrestrial climate. Clouds play an important role due to feedbacks of the radiation budget: variation of cloud cover/composition affects climate, which, in turn, affects cloud cover via atmospheric dynamics and sea temperature variations. Cloud formation and evolution are still under scientific scrutiny, since their microphysics is still not understood. Besides atmospheric dynamics and other internal climatic parameters, extraterrestrial sources of cloud cover variation are considered. One of these is the solar wind, whose effect on cloud cover might be modulated by the global atmospheric electrical circuit. Clouds height and composition, their seasonal variation and latitudinal distribution should be considered when trying to identify possible mechanisms by which solar energy is transferred to clouds. The influence of the solar wind on cloud formation can be assessed also through the $a_{p}$ index the geomagnetic storm index, which can be readily connected with interplanetary magnetic field, IMF structure. This paper proposes to assess the possible relationship between both cloud cover and solar wind proxies, as the $a_{p}$ index, function of cloud height and composition and also through seasonal studies. The data covers almost three solar cycles (1984-2009). Mechanisms are looked for by investigating observed trends or correlation at local/seasonal scale
\end{abstract}

\section{Introduction}

The anthropogenic activities are considered as the main drivers of climate change and of the heating of the terrestrial ecosystem. However, taking into account the complexity of the climate system, one has to consider natural climate drivers, such as the Sun or other extraterrestrial influences, such as the galactic cosmic rays [1-4].

It is well known that clouds have an important contribution to the radiative budget on Earth, contributing to climate variability. Cloud formation and their evolution are far from being completely understood $[5,6]$. 
Feedbacks of atmospheric dynamics and variations of humidity, air temperature, pressure distribution, and sea surface temperature presence contribute at various levels at the formation and life time of clouds. Extraterrestrial influences are thought to exist on clouds and mechanisms are based on modifying cloud condensation nuclei due to galactic cosmic ray induced ionization [7] and on modifications of cloud properties following changes in the global electrical circuit [8-12]. Changes in the solar wind, i.e. the flux of charged particles electrons and protons - coming out of the Sun with high speeds, have an important effect on ionospheric potential and atmospheric electricity [13]. Possible links between cloud cover and the interplanetary electric field have been found [12] and here we present results of our research aiming at identifying other solar wind parameters that might influence cloudiness.

The solar wind speed (SWS) is an important solar wind parameter, since it responds to solar activity changes on shorter or longer timescales [14]. Thus, correlations between the solar wind speed and cloud cover have been investigated at annual and seasonal scales. Since mechanisms of energy transfer from the solar wind to cloud cover involve processes at microphysics level, dependence of the correlation on cloud composition has been also studied.

Another solar wind parameter is the interplanetary magnetic field (IMF), whose influence on pressure centres and atmospheric dynamics has already been shown [15]. Besides the measure of the magnetic field, the geomagnetic storm index, $a_{p}$ influence on clouds and cloudiness is also assessed. The $a_{p}$ index is considered a good indicator of the daily geomagnetic activity at global level and is a measure of geomagnetic field fluctuations, giving, thus, information on the ionospheric perturbations as well [16,17]. The aim is to identify possible mechanisms through which solar energy may be transmitted to clouds.

\section{Data and methods}

The study has been conducted for low, middle and high clouds given by the ISCCP project (International Satellite Cloud Climatology Project), distinguished by their top pressure [18]. If existing, solar effects on clouds vary greatly with their height and geographic location [19-21]. Also, cloud microphysics strongly depends on cloud composition (i.e. liquid, ice or mixture: liquid+ice, as typically for middle clouds) and, in turn, cloud formation mechanism dictate their composition. Thus, we also distinguish between liquid and ice particles, thus 7 types of clouds are used in our study: low cloud amount (LCA) and middle cloud amount (MCA), regardless of their composition, high cloud amount (HCA) - which is composed only by ice, liquid low cloud amount (LLC), ice low cloud amount (ILC), liquid middle cloud amount (LMC) and ice middle cloud amount (IMC), respectively. All these 
cloud data sets, differentiated by cloud altitude and cloud composition, on a monthly basis, were downloaded from the ISCCP website [22] and were averaged for $5 \times 5 \mathrm{deg}$. grids all over the Earth.

The time frame under study covered 26 years, between 1984 and 2009, as they are the only whole years whose nebulosity is recorded within the ISCCP project (International Satellite Cloud Climatology Project). Data for SWS and $a_{p}$ index were found on the OMNIweb site of NASA [23], as daily datasets, from which monthly averages were computed. The temporal series for both cloud cover and solar wind parameters showed that trends exist, mainly for cloud covers, whose causes might be natural, but they also might be due to some instrumental errors on board of the monitoring sattelites [24]. Thus, in order to avoid possible artefacts, trends have been removed and data were detrended, since trends might be masking true correlations or induce correlations among different data series with no real, phenomenological support or reason $[25,26]$.

Data detrending was performed and correlation maps were built between each cloud type belonging to each of the 7 types, on one side, and SWS, and $a_{p}$, on the other side. The correlations were assessed for a significance threshold of $90 \%$. Annual and seasonal averages were used for better identifying the mechanisms between clouds and solar wind proxies. The seasons are defined with respect to the Northern Emisphere, such as spring comprises data for March, April and May, summer is June, July and August, autumn for September, October and November and winter for December (previous year), January and February.

\section{Results and discussion}

Correlation maps between the solar wind speed and cloud cover, using 12-months averages are given in Figs. 1, 2 and 3a, for low, middle and high clouds. Correlation coefficients, significant at $\mathrm{p}>90 \%$, are shown in colour code.

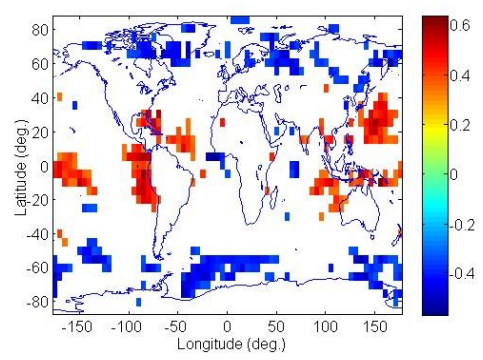

a) LCA - SWS

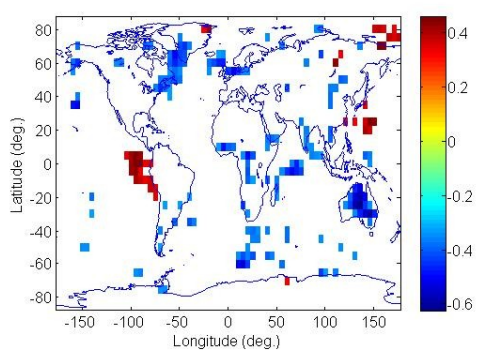

b) $\mathrm{LLC}-\mathrm{SWS}$

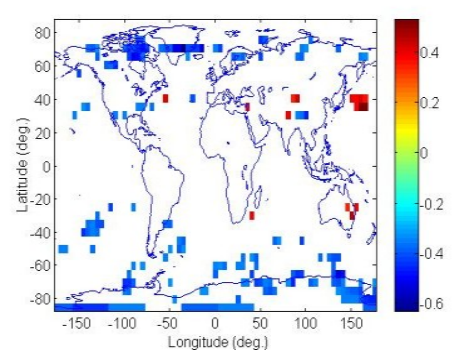

c) ILC - SWS

Fig. 1. Maps of significant correlation between low clouds and SWS for total (a), liquid and ice low clouds (b and c). Correlation coefficients are given in color coding (see color bars) and white areas correspond to low significance/no correlation 
Correlation is poor for high clouds, while low and middle clouds are positively correlated with SWS in a more consistent way. Regions where negative correlations exist are few and smaller. Middle and high clouds vary in phase with SWS above the Pacific Ocean, west of the Peruvian coast. Low clouds are anticorrelated with SWS in the same area. This specific area of strong correlations can be due or at least it can be strongly related to the El Nino or La Nina local conditions, which often develop in this region.

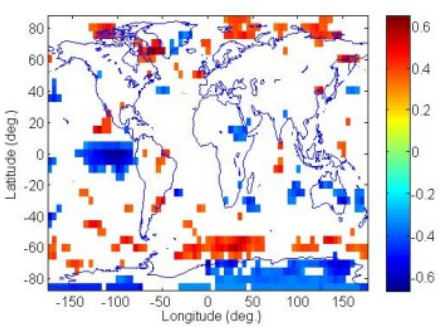

a) $\mathrm{MCA}-\mathrm{SWS}$

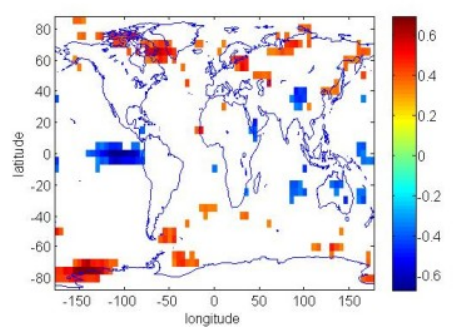

b) LMC - SWS

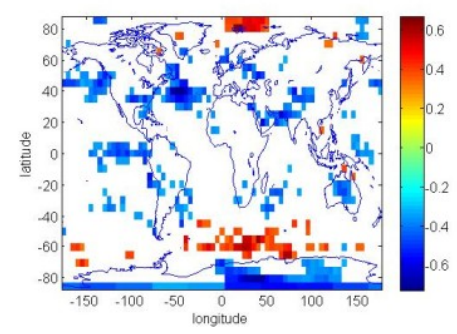

c) IMC - SWS

Fig. 2. Similar as Fig.1, but for middle clouds.

The separation between ice and water for low clouds brings no improvement in understanding possible mechanism since correlation maps shown in Figs. 1 (b, c) show only poor correlations with SWS. The correlation between liquid and, respectively, ice middle clouds with SWS, shown in Fig. $2 \mathrm{~b}$ and $\mathrm{c}$ is less defined as compared to total middle clouds. The alternating bands of positive/negative correlation in the Southern Hemisphere observed for middle clouds exist also when only ice clouds are considered. The basic problem when analysing middle clouds observed from satellite is their possible superposition with low or high clouds. High clouds, made only of ice do not correlate with SWS, except for the equatorial band observed in the Pacific for middle clouds, where ENSO plays a major role in atmospheric dynamics and cloud formation.

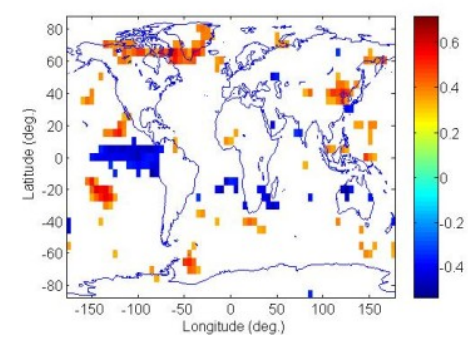

a) $\mathrm{HCA}-\mathrm{SWS}$

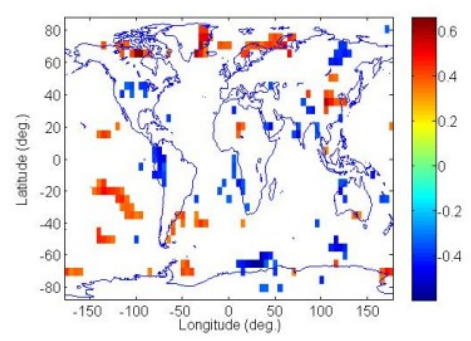

b) $\mathrm{HCA}-\mathrm{a}_{\mathrm{p}}$

Fig. 3. Significant correlation map between high clouds and: a) the solar wind speed; b) the geomagnetic storm index, $a_{p}$, 
Significant correlations between cloud cover and the geomagnetic storm index, $a_{p}$ were calculated using altitude separation and composition separation for each cloud type, using the same cloud data. Figs. 3, 4 and 5 present the results of the correlation analysis. The confidence level of the correlation is higher than $90 \%$.

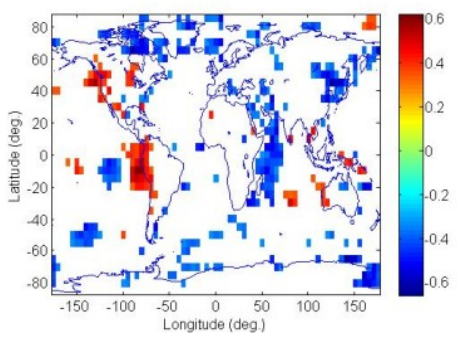

a) $\mathrm{LCA}-\mathrm{a}_{\mathrm{p}}$

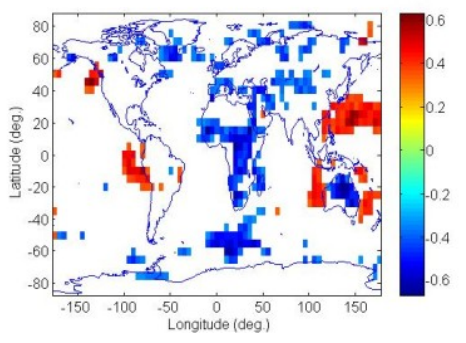

b) $\mathrm{LLC}-\mathrm{a}_{\mathrm{p}}$

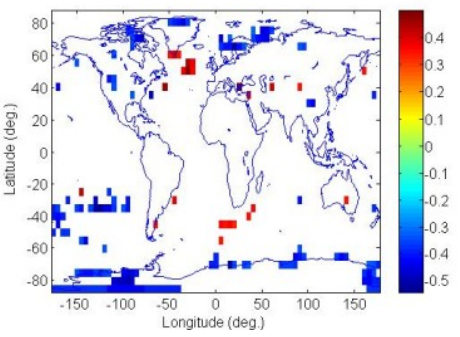

c) ILC $-a_{p}$

Fig. 4. Significant correlation maps between low clouds and the geomagnetic storm index, $a_{p}$, the clouds being differentiated by their composition (figs. b and c) and for the total low clouds (fig. a).

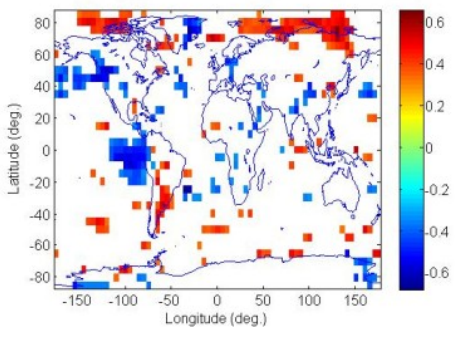

(a) $\mathrm{MCA}-\mathrm{a}_{\mathrm{p}}$

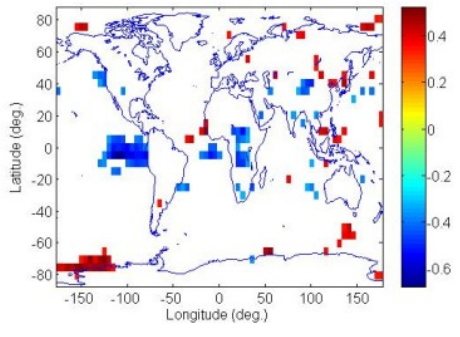

(b) $\mathrm{LMC}-\mathrm{a}_{\mathrm{p}}$

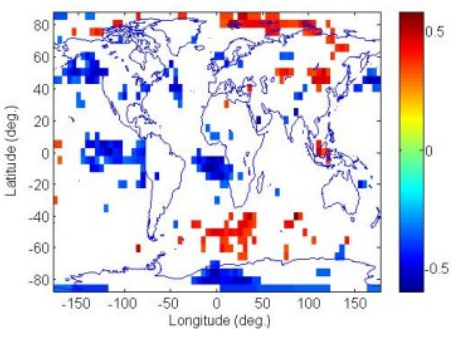

(c) $\mathrm{IMC}-\mathrm{a}_{\mathrm{p}}$

Fig. 5. Significant correlation maps between middle clouds and the geomagnetic storm index, $a_{p}$, for total middle clouds (a) and for clouds being differentiated by their composition ( $b$ and c).

The geomagnetic storm index correlates better with low clouds (LCA) than with middle or high clouds, in terms if area size. High clouds are not affected by geomagnetic storms, since any possible link would show up in a correlation. This shows that solar wind effects, if existing, depend on the altitudine and, possibly, on dominant composition (i.e. low clouds are mainly made of water, while middle clouds have mixed compositions). Correlations are found above the same area of the Peruvian coast/equatorial Pacific. This is seen even for high clouds, although at a smaller scale. This peculiar, yet consistent correlation area might indicate that El Nino/La Nina events might play a role. The middle and right-hand plots in Figs. 4 and 5 also show correlation maps for the two possible compositions: water and, respectively, ice low and for middle clouds. The correlation with $\mathrm{a}_{\mathrm{p}}$ is pretty good for water clouds and even improves compared to total low clouds. This points to a stronger influence of the geomagnetic activity on the low clouds made of water, thus the possible mechanism by which the geomagnetic activity might affect cloud cover should be searched in associated effects on water clouds. Low clouds made of water (LLC) are strongly anticorrelated with the 
$a_{p}$ index above a large part of Africa's South-Werstern shore, while in a large area above Eastern Asia, more specifically above the Japanese Archipelago, LLC are positively correlated with $\mathrm{a}_{\mathrm{p}}$, probably also through the mediation of different types of oceanic currents, coupled with the troposphere. The reason for a much lower correlation between low clouds made of ice (ILC) with $a_{p}$ might also be due to the very low percentage of ILC. Middle clouds made of water (LMC) correlate with $a_{p}$ sporadically, mainly above the Peruvian coast, while ice middle clouds (IMC) show a somewhat better correlation with the geomagnetic storm index, which looks closer to the total middle clouds, especially at middle-high latitudes.

Correlation areas between the $\mathrm{a}_{\mathrm{p}}$ index, on one side and low, middle and high clouds, respectively, on the other side, were compared to correlation maps between the galactic cosmic rays (CR) and cloud cover found in [19]. These comparisons aimed to identify in a different manner whether there are links between the variations of the terrestrial cloudiness and the variability of the interplanetary magnetic field (IMF), expressed through the geomagnetic storm index, $a_{p}$, which is directly related to IMF. Some superposition of correlation areas exist for low and middle clouds. This is expected, since a quiet geomagnetic period (low $\mathrm{a}_{\mathrm{p}}$ ) is accompanied by an increase in the CR flux and associated CR induced ionization, further supposed to increase cloud cover via increased nucleation rate. However, the coincidence is far from being perfect, suggesting that the proposed mechanism of CRII affecting low clouds is more complicated.

A seasonal analysis of the dependence of clouds-SWS and clouds- $\mathrm{a}_{\mathrm{p}}$ index correlations was also performed, the most significant results being shown in Figs. 6 and 7. No differentiation was done according to ice/water particles, since correlation does not seem to be affected by composition.

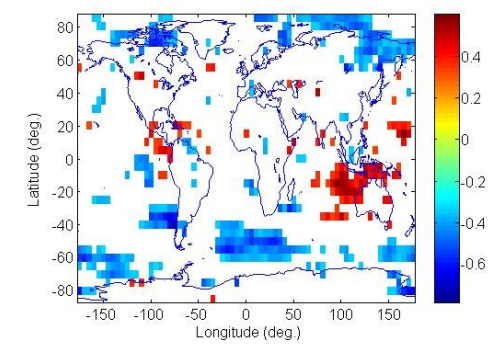

(a) LCA - SWS_autumn

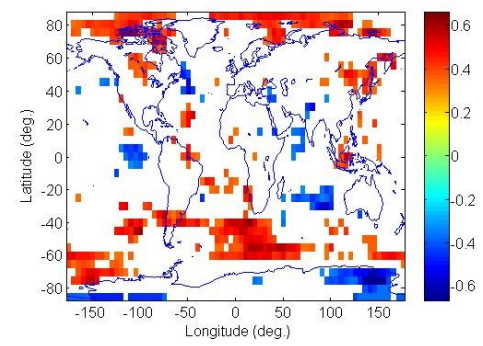

(b) MCA - SWS_autumn

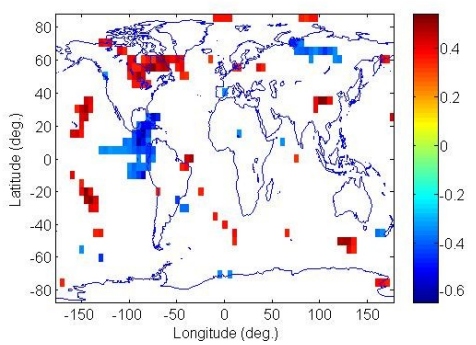

(c) HCA - SWS_winter

Fig. 6. Relevant seasonal correlation maps between SWS and clouds, as differentiated by altitudes, between 1984 and 2009.

Low and middle clouds correlate with SWS during autumn above approximately the same regions. The correlations are opposite for the two types of clouds, i.e. low cloud cover 
correlate negatively with SWS during autumns, except for the Eastern part of the Indian Ocean, while middle cloud cover positively correlate with SWS, and negative correlation is found above the Indian Ocean. Strong positive correlation areas between MCA and SWS are important for mid-high latitudes in the Southern Hemishphere (i.e. during local spring) and at high-polar latitudes in the Nothern hemisphere. For high clouds, some correlation with SWS is seen during the boreal winter but the areas are small and few.

Fig. 7 presents the best correlation maps when seasonal variation is considered. The best season for correlation is, again, autumn for low and middle clouds, similarly to the correlation with SWS. High clouds correlate with $a_{p}$ in winter. However, the correlations are poorer when $a_{p}$ is considered as compared to SWS. This is unexpected, since $a_{p}$ is seasonally dependent.

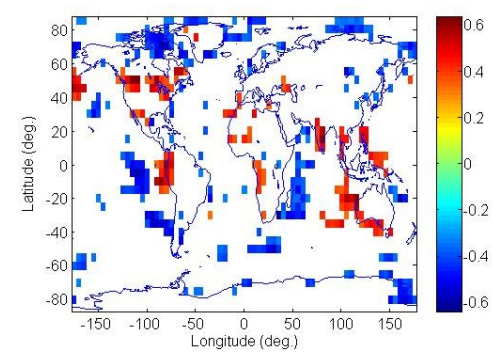

(a) LCA - ap_autumn

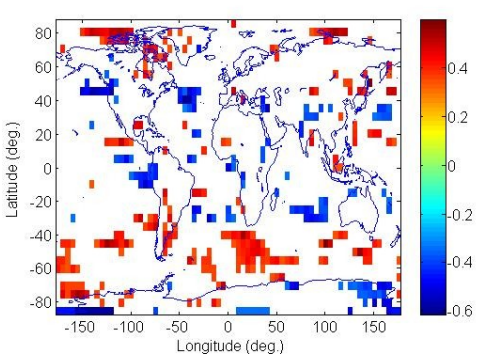

(b) MCA - ap_autumn

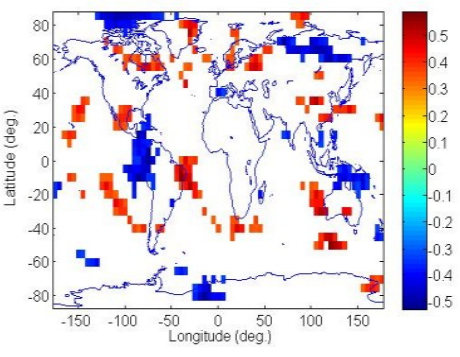

(c) HCA - ap_winter

Fig. 7. Relevant seasonal correlation maps between the geomagnetic storm index, ap and clouds, as differentiated by altitude, between $1984-2009$.

\section{Conclusions}

Possible correlations between cloud cover, on the one hand, and solar wind speed (SWS) and geomagnetic storm index, $\mathrm{a}_{\mathrm{p}}$, on the other hand were studied. The analysis was performed using detrended data, for the time span 1984-2009. The clouds were differentiated by altitude and composition. Seasonal variation was also studied.

Middle (MCA) and low clouds (LCA) correlate with SWS. Almost no correlation could be found between SWS and high clouds (HCA). Middle and high clouds are in phase with SWS, while low clouds are in antiphase with SWS over the ENSO region, i. e. West of the Peru's coast. No conclusion could be drawn regarding the correlation between SWS and water.ice clouds. Water clouds, however, seem to respond better to solar signals, while ice clouds are affected by SWS mainly at high latitudes. 
Low clouds are significantly correlated with the geomagnetic storm index on larger areas than middle and high clouds. Even though LCA is dominated by the liquid phase, the LLC-ap correlations are not entirely similar with total LCA-ap correlation distribution, the differences being highly visible over Africa, also easterly from China, in the Pacific Ocean and also at the southern Africa, while ILC has a specific pattern with respect to $a_{p}$, with scarce anticorrelation small regions - mainly over Antarctica.

There is no clear seasonal variation of the cloud cover - SWS correlation. Correlations are missing for all seasons except autumn for low and middle clouds and winter for high clouds. The responses of clouds to the variations of SWS and of $a_{p}$ depend on cloud composition and altitude, but much less on season.

Further investigations is required, in order to identify possible mchanisms of solar influence on clouds. ISCCP data might be appropriate for low, middle and high cloud studies, although problems have been discussed about the reliability of cloud data. However, possible artefacts have been shown to be small and cannot affect the entire globe and all types of cloud cover, at monthly and annual scale. The lack of correlation during seasons might imply that mechanisms that transfer solar energy to cloud cover have been dominated by local processes but might add up at longer scale.

\section{Acknowledgments}

This work was supported by a grant of the Romanian National Authority for Scientific Research, CNCS-UEFISCDI, project number PN-II-ID-PCE-2011-3-0709 (SOLACE/283). The ISCCP project and NASA's Omniweb databases are acknowledged for the data.

\section{References}

[1]L. J. Gray, J. Beer, M. Geller, J. D. Haigh, M. Lockwood, K. Matthes, U. Cubasch, D. Fleitmann, G. Harrison, L. Hood, J. Luterbacher, G. A. Meehl, D. Shindell, B. van Geel and W. White, Rev. Geophys. 48 (2010) RG4001, 1-53

[2]J. D. Haigh, A. R. Winning, R. Toumi, J. W. Harder, Nature 467 (2010) 696-699.

[3]M. Voiculescu, I. G. Usoskin and K. Mursula, Geophys. Res. Lett. 33 (2006) L21802, 1-5

[4]V. Ramanathan, R. D. Cess, E. F. Harrison, P. Minnis, B. R. Barkstrom, E. Ahmad and D. Hartmann, Science 243 (4887) (1989) 57-63

[5]H. Okamoto, AIP Conference Proceedings, 1531 (2013) 19

[6]D. Costa and O. Mares, AIP Conference Proceedings 1634 (2014) 151

[7]I. G. Usoskin, N. Marsh, G. A. Kovaltsov, K. Mursula, and O. G. Gladysheva, Geophys. Res. Lett. 31(2004b) L16109 
[8]B. A. Tinsley and F. Yu, Atmospheric ionization and clouds as links between solar activity and climate, AGU Monograph: Solar variability and its effect on climate 141 (2004) 321339

[9]S. Kinne, E. Raschke and M. Wild, AIP Conference Proceedings 1100 (2009) 549

[11]H. Deneke, R. Roebeling, E. Wolters, A. Feijt and C. Simmer, AIP Conference Proceedings 1100 (2009) 376

[12] M. J. Rycroft, K. A. Nicoll, K. L. Aplin and R. G. Harrison, J. Atmos. Sol.-Terr. Phy. 90-91 (2012) 198-211

[13] M. Voiculescu, I. G. Usoskin and S. Condurache-Bota, Environ. Res. Lett. 8, (2013) 045032

[14] D. Siingh D, R. P. Singh, A. K. Kamra, P. N. Gupta, R. Singh, V. Gopalakrishnan and A. K. Singh, J. Atmos. Sol.-Terr. Phys. 67 (2005) 637-658

[15] N. Marsh and H. Svensmark, Space Sci. Rev. 107(1-2), (2003) 317-325

[16]B. A. Tinsley, Space Sci. Rev. 94 (2000) 231-258

[17]I. Poole, Understanding Solar Indices, QST - Official Journal of ARRL, The National Association for Amateur Radio, 38-40, September (2002).

[18] http://www.ngdc.noaa.gov/stp/geomag/kp_ap.html

[19] W. B. Rossow and R. A. Schiffer, B. Am. Meteorol. Soc. 80 (1999) 2261-2287

[20] M. Voiculescu, I. G. Usoskin, Environ. Res. Lett. 7, (2012) 044004 (11pp)

[21] M. Voiculescu, I. G. Usoskin and K. Mursula, Geophys. Res. Lett. 33 (2006) L21802

[22] M. Voiculescu, I. G. Usoskin and K. Mursula, Adv. Space Res. 40 (2007) 1140-5

[23] http://isccp.giss.nasa.gov/products/browsed2.html

[24] http://omniweb.gsfc.nasa.gov/form/dx1.html

[25] A.T. Evan, A. K. Heidinger and D. J. Vimont, Geophys. Res. Lett. 34 (2007) L04701

[26] J. E. Kristjansson, J. Kristiansen and E. Kaas, Adv. Space Res. 34 (2004) 407-415.

[27] Z. Wu, N. E. Huang, S. R. Long and C.-K. Peng, PNAS - Proc. Nat. Acad. Sci. USA 104 (38) (2007) 14889-14894 